\title{
Análisis de señales para la detección de armónicos basado en el espacio complejo
}

\section{Signal analysis to harmonics detection based in complex space}

\author{
CONTRERAS-HERNÁNDEZ, Jose L. †*, ALMANZA-OJEDA, Dora L. y IBARRA-MANZANO \\ Mario A.
}

Universidad de Guanajuato

ID $1^{\text {er }}$ Autor: José, Contreras-Hernández / ORC ID: 0000-0003-0405-5554 CVU: 487660

ID 1 ${ }^{\text {er }}$ Coautor: Dora, Almanza-Ojeda / ORC ID: 0000-0002-3373-0929 CVU: 50006

ID $2^{\text {do }}$ Coautor: Mario, Ibarra-Manzano / ORC ID: 0000-0003-4317-0248 CVU: 105633

DOI: $10.35429 / J C S .2019 .7 .3 .1 .4$

Recibido: 12 de Enero, 2019; Aceptado 02 de Marzo, 2019

\begin{abstract}
Resumen
Este trabajo presenta la detección de armónicos mediante el análisis de señales en espacio complejo. Se inicia obteniendo la división punto a punto de la muestra de interés con la muestra anterior de las figuras de Lissajous generadas por dos señales en el espacio complejo. Después, con la señal obtenida de la división, se calcula el ángulo de cada elemento para analizar su comportamiento. Las características conseguidas proporcionan información numérica de las frecuencias que componen una señal. Esto simplifica el análisis sin necesidad de transformaciones u operaciones de alto costo computacional.
\end{abstract}

\begin{abstract}
This work presents harmonic detection through signal analysis in complex space. This method obtains the pointto-point división of the present sample by the last sample of the Lissajous curve which is generated by two signals in complex space. Then, the angle is calculated to each element to obtain the behavior. The obtained features provide numerical information of the frequencies that form a signal. This is obtained without space transformation or operation with high computational cost.
\end{abstract}

Lissajous, Complex space, Harmonics detection

Lissajous, Espacio complejo, Detección armónicos

Citation: CONTRERAS-HERNANDEZ, Jose L. $\dagger^{*}$, ALMANZA-OJEDA, Dora L. y IBARRA-MANZANO Mario A. Análisis de señales para la detección de armónicos basado en el espacio complejo. Revista de Simulación Computacional. 2019 3-7: 1-4

\footnotetext{
* Correspondencia al autor: jose.contreras@ugto.mx

$\dagger$ Investigador contribuyendo como primer autor.
} 


\section{Introducción}

Las señales armónicas se presentan en maquinaria de combustión interna, líneas eléctricas o instrumentos musicales y las cuales nos proporcionan características de los distintos sistemas que las producen [1]. Las frecuencias que componen este tipo de señales obtienen por medio del procesamiento y análisis profundo de atributos. Entre los métodos más utilizados para la detección de armónicos en señales se encuentra la Transformada Rápida de Fourier (FTT) [2], métodos de clasificación como las Máquinas de Vectores de Soporte (SVM) [3] o Redes neuronales (ANN) [4] y los métodos basados en Wavelets [5].

En trabajos como el mostrado en [1], los métodos basados en FFT realizan la aproximación de la reconstrucción de la señal a analizar por medio de frecuencias discretas, lo cual conlleva un cambio de espacio y cálculos con una mayor carga computacional. El método desarrollado por SVM utiliza funciones de ajuste para detectar los armónicos de las señales en sistemas eléctricos, sin embargo, el modelo y el pequeño muestreo llegan a afectar la precisión de sus componentes como se describe en [3]. De igual manera, las (ANN) son usadas para la detección de armónicos en redes eléctricas como lo describe [6], la cual presenta una mayor precisión con la dificultad de obtener una muestra ideal de entrenamiento, lo cual afecta la velocidad del método [7]. Como los autores describen en [5], los métodos basados en Wavelets para la detección de armónicos en señales de corriente han obtenido buenos resultados, en este trabajo, el esquema de elevación de daubechies 9/7 es aplicado para la detección de armónicos.

El trabajo aquí desarrollado propone el método de análisis geométrico. Éste se basa en la teoría de procesamiento algebraico de señales [8], en la cual la teoría algebraica hace la derivación de algoritmos de forma concisa y transparente, da una visión de su estructura, permite clasificar los existentes y descubrir nuevos para transformadas existentes y nuevas [9]. Se presenta el método para la obtención numérica de la cantidad de frecuencias que componen a una señal por medio de operaciones de bajo costo computacional en números complejos.
Este trabajo se divide en 3 secciones. En la Metodología se presenta el desarrollo matemático utilizado para la obtención de las componentes en frecuencia de una señal. En Resultados se presentan las señales que se probarán y el resultado del método. Finalmente, en la última sección se presentan las conclusiones de este trabajo y trabajo futuro.

\section{Metodología}

La figura de Lissajous es una herramienta que permite analizar e interpretar la interacción correspondiente a la superposición de dos señales con movimientos armónicos simples en direcciones perpendiculares. En este trabajo se presentan las señales seno y coseno descritas por las ecuaciones (1) y (2) de la forma

$$
\begin{aligned}
& x(k)=\sin (k t) \\
& y(k)=\cos (k t) .
\end{aligned}
$$

El comportamiento de las señales $x(k)$ y $y(k)$ es mostrado en la Figura 1a) con un periodo de 0 a $2 \pi$ y amplitud unitaria. Estas señales pueden ser expresadas en espacio de los números complejos de la forma que se muestra en la ecuación siguiente

$$
z(k)=x(k)+y(k) i
$$

El resultado de representar ambas señales en el espacio complejo corresponde a las figuras de Lissajous como se muestra en la Figura 1b). Partiendo de este tipo de representación, la apariencia de la figura creada por estas dos señales es sensible a los valores de frecuencia, amplitud, fase y offset, como se puede observar en la Figura 2, en la que se muestran las figuras de Lissajous resultantes al variar la amplitud de la señal en $y$, por lo que su análisis matemático permite determinar si alguna de sus características ha sido modificada [10].

Una opción en este tipo de análisis es la obtención de la componente $z_{c}$ la cual, al ser multiplicada por $z(k)$, permite la obtención de $z(k+1)$. El valor $z_{c}(k)$ se consigue por medio de la división punto a punto de la parte real e imaginaria de $z(k+1)$ entre $z(k)$ señalada en la ecuación (4)

$$
z_{c}(k)=\frac{\operatorname{real}(z(k+1))}{\operatorname{real}(z(k))}+\frac{\operatorname{imag}(z(k+1))}{\operatorname{imag}(z(k))} \mathrm{i}
$$




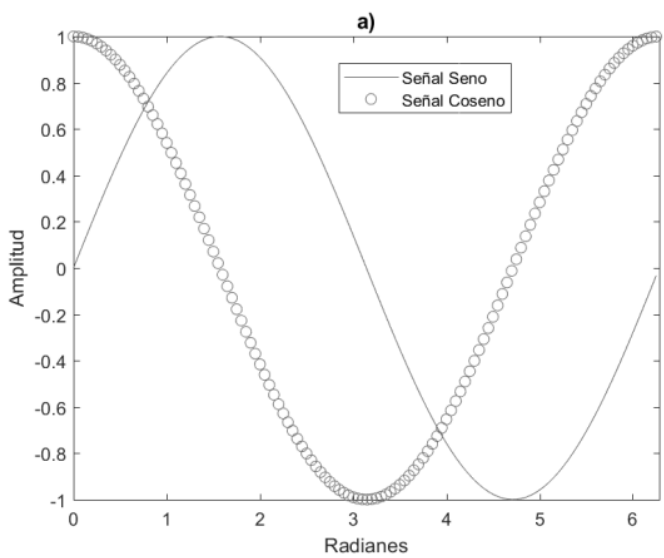

b)

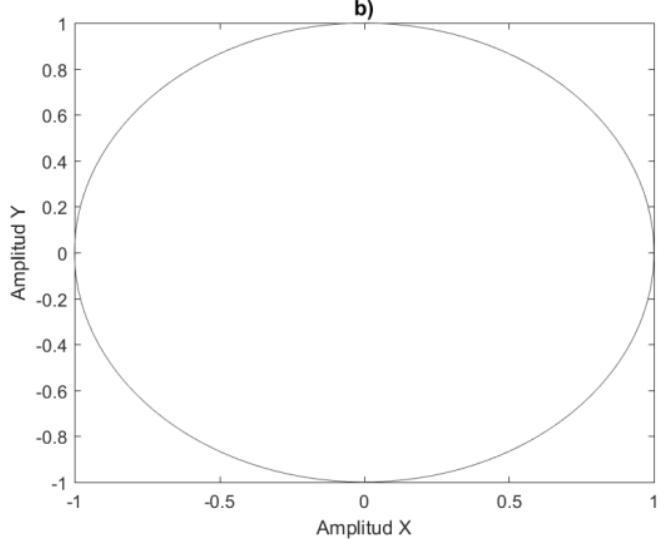

Figura 1 a) Señales x(k) y y(k). b) Gráfica de z(k)

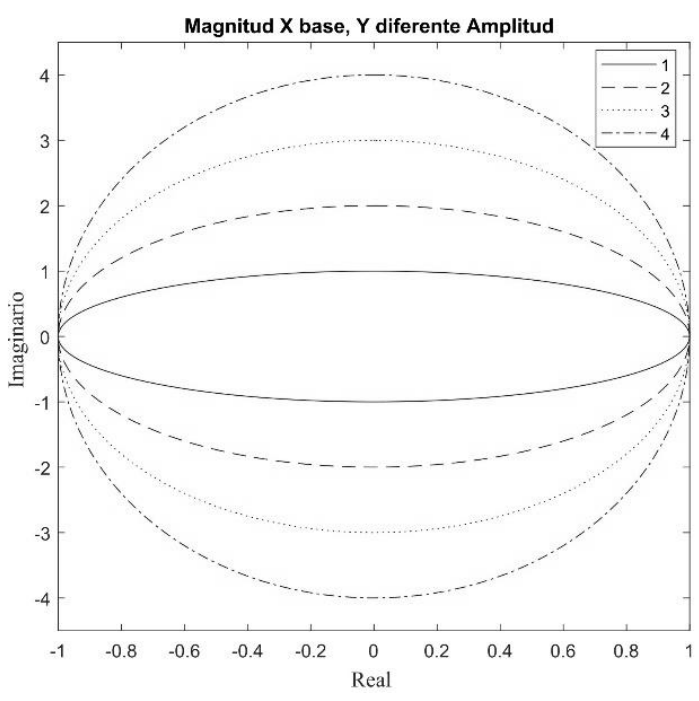

Figura 2 Gráficas de las figuras de Lissajous resultantes de la variación en la amplitud de la señal Y

Con la componente $z_{c}(k)$ calculada, se obtiene el ángulo $\theta_{c}(k)$ mediante

$$
\theta_{c}(k)=\frac{\operatorname{real}\left(z_{c}(k)\right)}{\operatorname{imag}\left(z_{c}(k)\right)}
$$

Analizando el comportamiento de este ángulo, se consiguen características de frecuencia en las señales, tal es el caso de los armónicos.
Con el fin de comprobar el método aquí presentado, a las señales originales se les aplica la suma de armónicos de acuerdo con las ecuaciones

$$
\begin{aligned}
& x_{a}(k)=\sin (k t)+\sin (2 k t)+\sin (3 k t) \ldots \sin (n k t) \\
& y_{a}(k)=\cos (k t)+\cos (2 k t)+\cos (3 k t) \ldots \cos (n k t)
\end{aligned}
$$

Las pruebas realizadas constan de la suma de armónicos desde $n=1$ hasta $n=5$ para las señales $X \quad$ y $Y$. Las señales armónicas desarrolladas se muestran en la Figura 3. Así mismo, las figuras de Lissajous trazadas a partir de estas señales se muestran en la Figura 4.

\section{Resultados}

Después de obtener las señales con la suma de armónicos y las cuales generan una figura de Lissajous expresada en el espacio de números complejos, se calculan los ángulos de las componentes $z_{c}(k)$ de cada una, dando como resultado las gráficas mostradas en la Figura 5. En esta figura se muestra que existe una relación de la suma de armónicos en las señales con respecto a los picos que se presentan. Si se tiene una señal base sin armónico, el resultado es una recta. Si a esa señal se le suma su armónico, tanto al seno como en coseno, se presenta un pico en el ángulo formado en $z_{c}(k)$. Si a las señales a analizar se les suma su segundo armónico correspondiente, la gráfica resultante muestra dos picos y así sucesivamente. De esta manera se consiguen características de la suma de los armónicos correspondientes de dos señales calculando el ángulo de $z_{c}(k)$ de la figura de Lissajous representada en el espacio complejo.
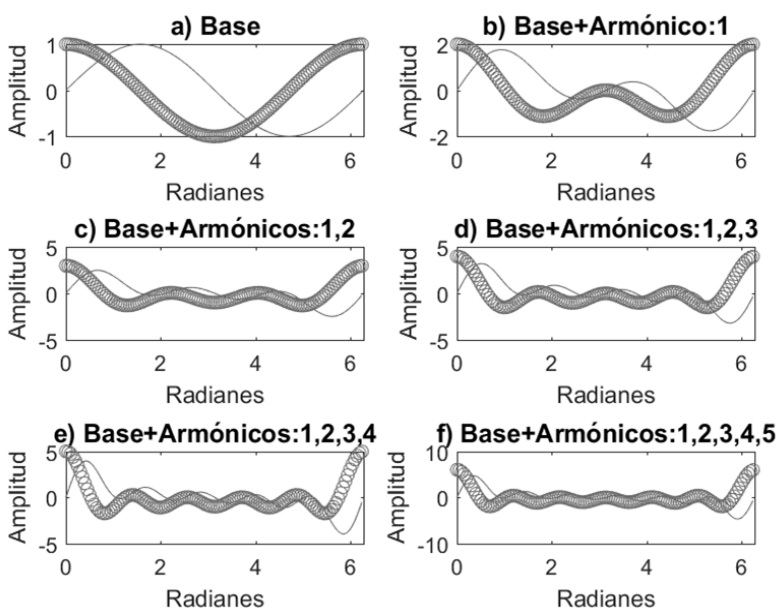

Figura 3 Gráficas de las señales resultantes de la suma de los armónicos de las señales seno (-) y coseno (o)

CONTRERAS-HERNANDEZ, Jose L. $\dagger^{*}$, ALMANZA-OJEDA, Dora L. y IBARRA-MANZANO Mario A. Análisis de señales para la detección de armónicos basado en el espacio complejo. Revista de Simulación Computacional. 2019 

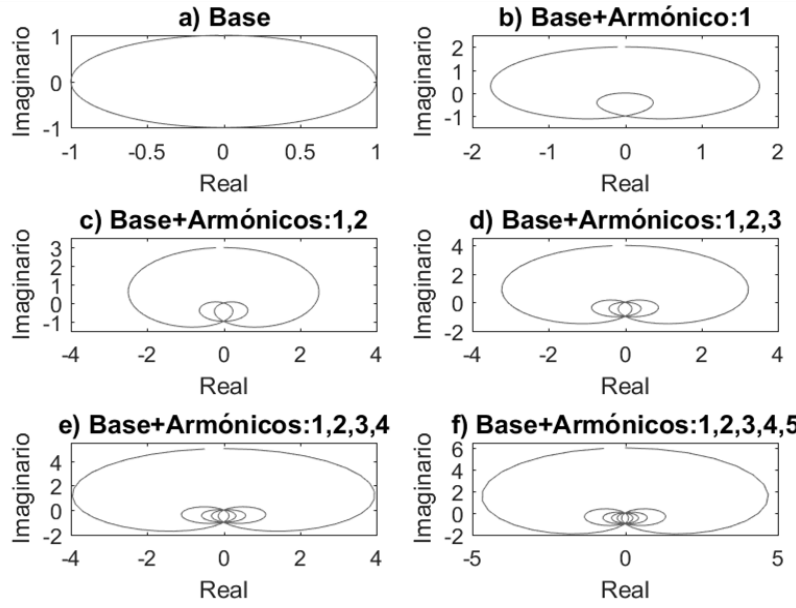

Figura 4 Gráficas de las figuras de Lissajous resultantes de la suma de los armónicos de las señales seno y coseno
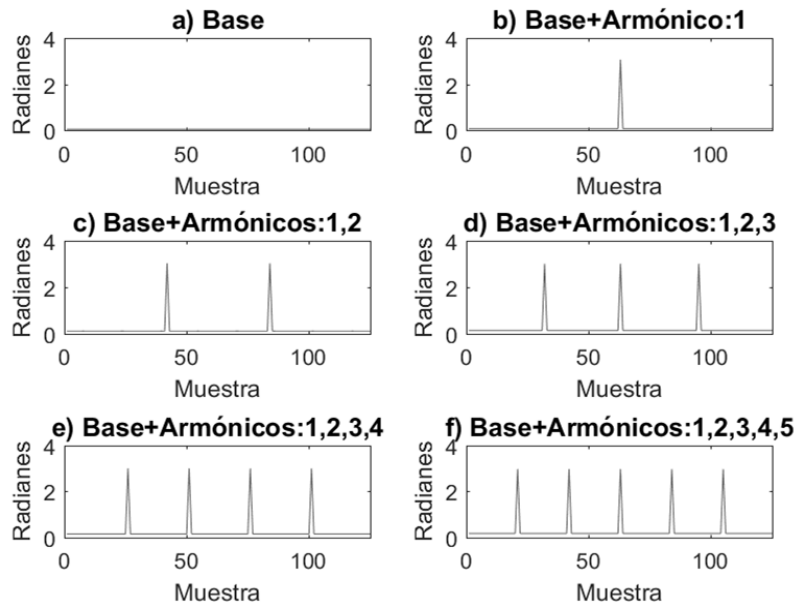

Figura 5 Gráficas de los ángulos calculados de las componentes de la suma de armónicos

\section{Conclusiones}

El análisis de las figuras de Lissajous en el espacio complejo es una herramienta muy útil. Mediante el cálculo del ángulo se extraen características de los armónicos de manera numérica representados por picos en las gráficas resultantes. El trabajo se desarrolló aplicando la suma de armónicos a las señales seno y coseno, por lo que es importante realizar pruebas tomando en cuenta las distintas variaciones y combinaciones que se tienen en las señales para extraer las características correspondientes de frecuencia, amplitud, fase y offset.

\section{Referencias}

[1] Piper, J. E. Reese, S. S. and Reese, W. G. (2012). Detection of harmonic signals. 2012 Oceans. Hampton Roads. 1-4.
[2] Zeytinoglu, M. and Wong, K. M. (Nov, 1995). Detection of harmonic sets. IEEE Trans on Signal Processing. (43). 2618-2630.

[3] Cao, J. Ling, T. Zhang, M. and Liu, L. (2008). Monitoring Method of Power System Interharmonics. High Voltage Engineering. (8). 1745-1750.

[4] Luo, H. and Luo, S. (Jan, 2011). The Reseach Summary of Harmonic Study In Electric Power System. China, Industrial Instrumentation and Automation. (5). 64-67.

[5] Wang, Y. Li, Z. and Guo, G. (2011). Research on methods of harmonic detection based on second generation wavelet algorithm. 2011 International Conference on Electric Information and Control Engineering. 60906093.

[6] Ma, X. Yin, Z. Zhou, L. and Zhu, Y. (2008). The research summary of harmonic detection in electric power system. China, Sichuan Electric Power Technology. (4). 53-56.

[7] Wei, W. Zengli, L. Lin, C. and Weiwei, S. (2013). Harmonic Detection of Power System Based on SVD and EMD. 2013 Ninth International Conference on Computational Intelligence and Security. 185-189.

[8] Fuhrmann, P. (2012) A polynomial approach to linear algebra. New York: Springer Press.

[9] Ran, T. Bing-zhao, L. and Hua-fei, S. (2013). Research Progress of The Algebraic and Geometric Signal Processing. (9). 40-47.

[10] Churchill, R.V. and Brown, J.W. (1992). Variable compleja y aplicaciones. McGraw Hill. Quinta Ed.

[11] French, A.P. (2003). Vibrations and Waves. CRC press. 198.

[12] Fuster R. and Giménez I. (2006). Variable compleja y ecuaciones diferenciales. Reverté. 